\title{
A Novel Research Technology to Explore the Mystery of Traditional Chinese Medicine: Optogenetics
}

\author{
Wei-Kang Luo, ${ }^{1}$ Dan Zhou, ${ }^{1}$ Jia-Miao Liu, ${ }^{2}$ Yang Hang, ${ }^{2}$ Zhao-Yu Yang, ${ }^{1}$ Yao Wu, \\ Liang-Lin Zhang, ${ }^{1}$ and Yang Wang ${ }^{1}$ \\ ${ }^{1}$ Institute of Integrative Medicine, Xiangya Hospital, Central South University, Changsha 410008, China \\ ${ }^{2}$ Xiangya School of Medicine, Central South University, Changsha 410013, China \\ Correspondence should be addressed to Yang Wang; wangyang_xy87@csu.edu.cn
}

Received 27 October 2020; Revised 5 April 2021; Accepted 10 April 2021; Published 20 April 2021

Academic Editor: Daniel Dias Rufino Arcanjo

Copyright ( 92021 Wei-Kang Luo et al. This is an open access article distributed under the Creative Commons Attribution License, which permits unrestricted use, distribution, and reproduction in any medium, provided the original work is properly cited.

Traditional Chinese medicine (TCM) is gaining increasing popularity worldwide for the function of health promotion and adjuvant therapy. However, the world's understanding of TCM is far from enough, which seriously limits the modernization and internationalization of TCM. Therefore, modern and efficient analytical methods are urgently needed to understand the mechanism of TCM. Optogenetics is one of the most prevalent technologies in the $21^{\text {st }}$ century and has been used to explore life science, especially neuroscience. It already has had great influences in the study of neural circuits and animal models of mental diseases and was named "Method of the Year" by the Nature Methods journal in 2010. Increased interests occurred in the applications of optogenetics to explore a myriad of medical and mental health disorders. However, it has not so far been noticed by TCM researchers. We elaborated on an idea that introducing this technique into the field of TCM research to improve diagnosis, treatments, and evaluating the therapeutic effects. In this review, we made a systematic prospect in the theory, feasibility, and application of TCM optogenetics. We mainly focused on applying optogenetic methodologies to make a more comprehensive understanding of TCM.

\section{Introduction}

Traditional Chinese medicine (TCM), as the core component of international complementary and alternative medicine (CAM), has a history of more than 8000 years in China [1]. Based on empirical knowledge and theories and concepts, TCM has influenced various other Asian medical systems and evolved as a unique system of medical practice $[2,3]$. In developed countries, the acceptance of CAM services has increased over the past decade, particularly massage. Data from the censuses of population indicated that Australia (52-69\% of those surveyed), Canada (59$60 \%)$, the United States (62\%), Singapore (76\%), and Japan $(50 \%)$ had used CAM [4]. Due to its unique holistic philosophy, therapeutic theories, and diagnostic methods, TCM has become the cultural treasure of the Chinese nation [5].

TCM mainly involves early intervention, combination therapies, and personalized medicine [6]. The traditional
Chinese herb, acupuncture, and other nonmedication therapies are the main research methods, which are good for improving health, preventing, and healing diseases. Compared with western medicine, TCM-induced effects are related to multiple factors and responsible for treating complex diseases because of multicomponent, multitarget, and multipathway [7]; TCM also has low toxicity and sideeffects; TCM-targeting preparation has unique advantages in the drug delivery system [8]. However, it has a huge gap between excellent clinical application and insufficient theory investigation. To be understood and accepted by the public and the medical system, TCM has widely investigated and provided more objective shreds of evidence, especially in China [9].

Entering the $21^{\text {st }}$ century, a new era for modernization of TCM was launched with the development of scientific technology. Plenty of evidence and research methods have been produced and updated $[10,11]$, including molecular 
imaging, molecular biology, evidence-based medicine, multivariate analyses, network modeling, "Big Data" research, gene differential expression technology, and multidisciplinary exploration. Unfortunately, owing to the complex targets and pathways, current methods can not completely elucidate the underlying mechanisms of action of TCM researches. Novel biotechnologies are urgently needed to meet the requirements of TCM investigation.

Optogenetics is an emerging way to leverage microbial opsins and molecular genetics to enable temporally precise control of genetically defined neurons in the mammalian brain in vivo with light [12]. Advances in optogenetics have increased exponentially in recent years. The Nature Methods and Science journal praised optogenetics as "Method of the Year" and "Breakthroughs of the Decade," respectively $[13,14]$. It has also made many breakthroughs in the past decade. By delivering optical control at the speed (millisecond-scale) and with the precision (cell type-specific) required for biological processing, optogenetic approaches have opened new landscapes for the study of biology in both health and diseases [15]. Although the explorations of this method in biomedicine are increasingly deep and mature, we have not found the relevant optogenetic researches in the field of TCM. Here, we proposed for the first time using optogenetics to uncover the mystery of TCM and its increased applications (Figure 1).

\section{Existing Approaches to TCM Research}

To unveil the efficacy mechanism of TCM, numerous up-todate scientific investments have been used in this field. Molecular imaging has been applied in TCM therapy for neurological disorders. It converts classical morphological measures into the assessment of functional, cellular, metabolic, and molecular information in vivo [6] and various other TCM therapies (herbs, formulations, acupuncture, moxibustion, massage, Gua Sha, and diet therapy [16]). Functional magnetic resonance imaging has become an important scientific tool to explore the underlying neural mechanisms of acupuncture [17]. Molecular biology has been widely used in the investigation of the bioactive compounds of Chinese herbs such as Rhizoma Paridis [18] and the molecular mechanisms of diagnosis and treatment of TCM [19]. In the field of evidence-based medicine, Chinese prescriptions, therapies, and dosages were tailored for each patient and assessed through the current standard method of randomized controlled clinical trials. Recently, associated high-throughput omics technologies are dramatically accelerating the pace to discover and analyze the mechanisms of action of herbal formulae and Chinese syndrome [20-22]. Multivariate analyses and network modeling enable researchers to comprehensively identify and evaluate the natural herb's diversity and functionality $[20,23]$. TCM network pharmacology methods were created to prioritize disease-associated genes, predict the target profiles and pharmacological actions of herbal compounds, reveal drug-gene-disease comodule associations, screen synergistic multicompounds from herbal formulae in a highthroughput manner, and interpret the combinatorial rules and network regulation effects of herbal formulae [24]. With the current globalization of life sciences and the arrival of "Big Data" research and development, TCM chemical databases, such as TCM Database@Taiwan [25] and HerbBioMap database, and some literature generally provide the main sources of information on the chemical profiles of TCM [26]. The emergence of gene differential expression technology provides a strong arm for TCM plant studies such as Viola yedoensis Makino [26]. This technology enables researchers to assay target-genetic differential expression after TCM treatment in a high-throughput manner [27]. TCM research integrated with other disciplines is progressing. Bioinformatics is used to analyze TCM data and provide biological evidence for the basic understanding of mechanisms, safety, and efficacy of TCM treatments [28]. The nanocarriers are applied to deliver the active ingredients or fractions of TCM [29]. The nonlinear dynamical complex systems (NDS) science informs future studies of the whole systems of TCM [30]. High-tech acupuncture is dedicated to the innovation of traditional acupuncture and the combination of traditional acupuncture and modern technologies. The existing robot-controlled acupuncture (RCA) [31], laser acupuncture [32], electroacupuncture [33, 34], and teleacupuncture [35-38] are all important directions of high-tech acupuncture research. The modernization of acupuncture using high-tech methods can accelerate the process of clinical acupuncture practice, obtain insight into the mechanism of acupuncture, and open up new dimensions in acupuncture researches. These methods are truly providing contemporary evidence to support TCM simplification (Table 1).

Despite the strengths of current technologies, we acknowledge potential limitations that should be considered. First, the uncontrollable quality is a bottleneck for the modernization and globalization of TCM [39]. For an up-todate database, the quality of TCM is always inaccurate because the chemical profiles are always changing. Second, the current pharmacological approaches are also limited by temporal resolution. They cannot achieve millisecond-scale genetically targeted optical control, which will result in the inability to observe each individual in time and accurately. Third, the mechanism of action in TCM is still unclear. Fourth, the modern connotation of a specific TCM Zheng has not been explained clearly, which is the key to TCM research.

\section{Theory of TCM Optogenetics}

Up to now, contemporary scientific studies of TCM have revealed only part of its value to global health. To be a wildly acceptable treatment, TCM needs a more robust and reliable technology to completely understand the deep theoretical foundations and clear determined mechanisms [40].

The optogenetic approach is a tool to manipulate cellular function by using optics and genetics to influence the transmembrane of ions [41]. The superiority of noninvasive and precisely targeted with exquisite spatial and temporal precision can report the presence or activity of specific molecular when stimulated by multiple wavelength light [42]. The essence of this technique is a process from 


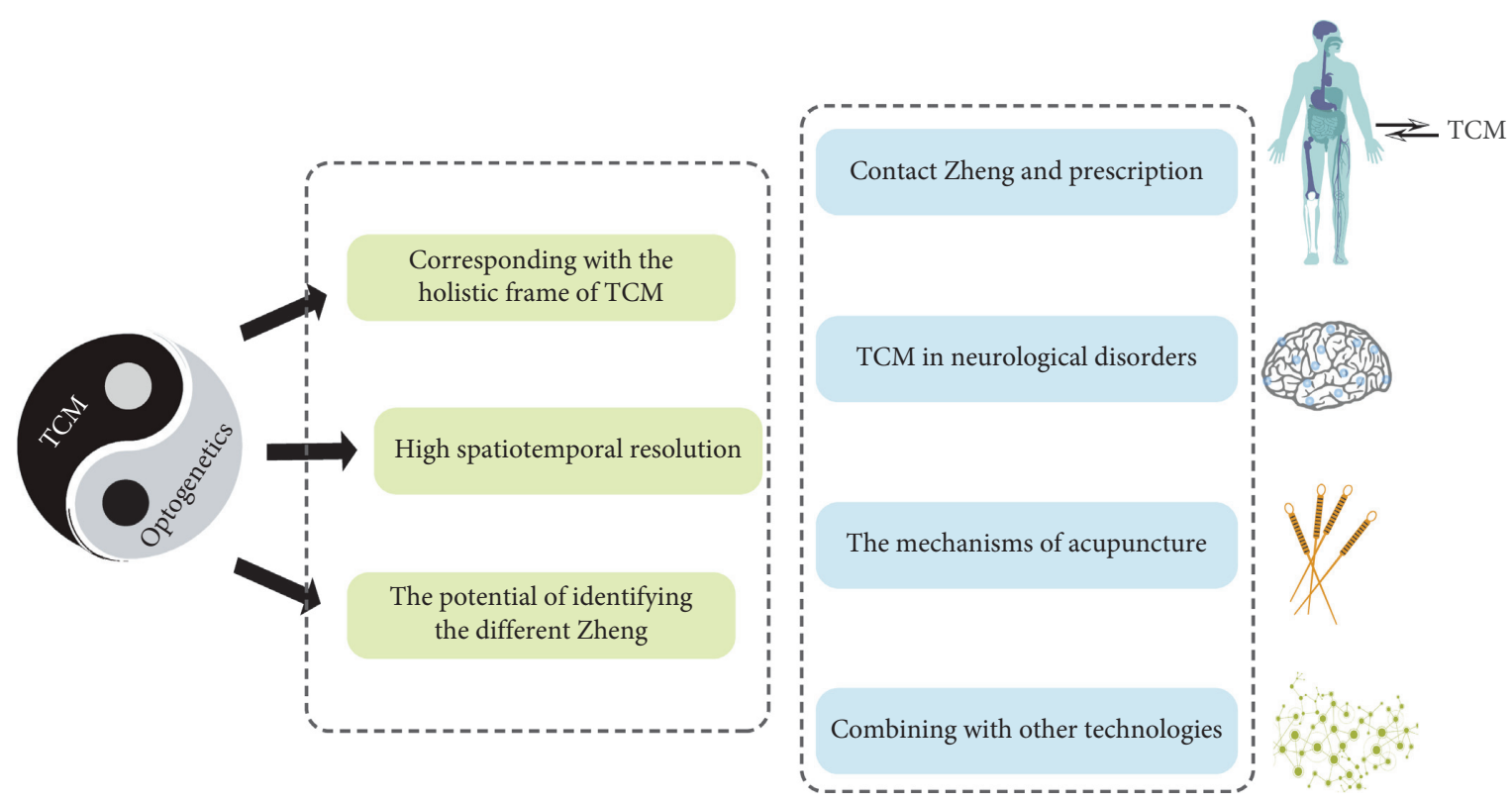

FIgURE 1: The theory, feasibility, and application of TCM optogenetics. TCM: traditional Chinese medicine.

TABLE 1: Selected modern research of TCM in the international journal.

\begin{tabular}{|c|c|c|c|}
\hline $\begin{array}{l}\text { Technique/ } \\
\text { method }\end{array}$ & Related research in TCM & Functional outcome & $\begin{array}{c}\text { Year/ } \\
\text { Ref }\end{array}$ \\
\hline $\begin{array}{l}\text { Molecular } \\
\text { imaging }\end{array}$ & $\begin{array}{c}\text { Neurological diseases } \\
\text { (MCAO, AD, PD, Stroke) }\end{array}$ & Monitoring the function of diseases & 2013 \\
\hline $\begin{array}{l}\text { Molecular } \\
\text { biology }\end{array}$ & Chinese herb (Rhizoma Paridis) & Assessed the effects of activity component in disease & 2015 \\
\hline RCT & $\begin{array}{l}\text { Chinese prescription/herb(Qili Qiangxin } \\
\text { Capsules, Xuezhikang) }\end{array}$ & Entered the FDA clinical trial in the United States & $\begin{array}{l}2013 \\
2014\end{array}$ \\
\hline Omics & TCM Zheng & $\begin{array}{c}\text { Offered the long-standing clinical and phenotypic-rich } \\
\text { insights at the same time }\end{array}$ & 2013 \\
\hline $\begin{array}{l}\text { Network } \\
\text { pharmacology }\end{array}$ & Chinese herb & $\begin{array}{l}\text { Revealed the combinatorial rules and network regulation } \\
\text { effects of herbal formulae }\end{array}$ & 2013 \\
\hline Big data & TCM Database@Taiwan & $\begin{array}{c}\text { Provided the main sources of information on the chemical } \\
\text { profiles of TCM }\end{array}$ & 2011 \\
\hline GDET & $\begin{array}{l}\text { Chinese traditional medicine plant (Viola } \\
\text { yedoensis Makino) }\end{array}$ & $\begin{array}{l}\text { Enabled to assay target-genetic differential expression after } \\
\text { TCM treatment in a high-throughput manner }\end{array}$ & 2015 \\
\hline Nanocarriers & Absorptive method of TCM & Delivered the active TCM ingredients or fractions & 2015 \\
\hline NDS & Models and methods research of TCM & $\begin{array}{l}\text { Characterized different constitutional types and document } \\
\text { the evolution and dynamics of whole-person healing and } \\
\text { well-being over time }\end{array}$ & 2012 \\
\hline $\begin{array}{l}\text { High-tech } \\
\text { acupuncture }\end{array}$ & $\begin{array}{l}\text { Traditional acupuncture technique (RCA, } \\
\text { RAA, electroacupuncture, laser acupuncture) }\end{array}$ & $\begin{array}{l}\text { Helped clinical acupuncture practice to progress and prove } \\
\text { some fundamental principles of traditional acupuncture } \\
\text { practice }\end{array}$ & 2009 \\
\hline
\end{tabular}

Source: MCAO, middle cerebral artery occlusion; AD, Alzheimer's disease; PD, Parkinson's disease; NDS, nonlinear dynamical complex systems; RCT, randomized controlled clinical trials; GDET, gene differential expression technology; RCA, robot-controlled acupuncture; RAA, robot-assisted acupuncture.

microcosm moved on to holism. In the research of optogenetics and depression, the optogenetic tool revealed the projection pathway network of neural via stimulating or inhibiting dopamine neurons in the ventral tegmental area (VTA) and bidirectional mediate depression-related behavior [43], while TCM would treat the depression by grasping the Chinese syndrome on the macrography transformed to manipulate each of the subtle parts through Chinese prescription. Any vital movement of organisms was comprised of the function of every single cell. The form of entirety and individual is indivisible. Hence, optogenetics is the best theoretical fit between the positive influence of TCM from the whole to the individual and the reverse study of optogenetics from the individual to the whole. It will become an important bond to combine modern science and TCM. This pattern integrating modern optogenetics into TCM may be applied more widely. 
Theoretically, TCM optogenetics may possess three major characteristics: accuracy, simplification, and predictability. The optogenetics will clarify the specific TCM diagnosis and treatment mechanism through exploring the actions of drugs, herbal formulae, acupuncture, and Chinese syndromes and their interrelation, eventually to guide the clinic. Ultimately, the pattern of TCM optogenetics may be built like this: the basic theory of integrating the idea of optogenetics and the philosophy of TCM; the generation, assessment, and application of TCM optogenetics evidence naming pattern; then, the efficacy which needs combining the optogenetic technique and Chinese individualized treatment to inspect the long-term impact.

\section{The Feasibility of TCM Optogenetics}

The TCM optogenetic approach can provide a new research paradigm for transforming TCM from experience-based medicine to an evidence-based medicine system. We will elaborate on the feasibility of TCM optogenetics in the following aspects.

4.1. Corresponding with the Holistic Frame of TCM in the Brain. Optogenetics includes the use of a variety of genetically encoded probes for physiological parameters ranging from membrane voltage and calcium concentration to metabolism [44]. The key factor for optogenetics is the photosensitive protein. Spatiotemporal precision of neural control was achieved by an optogenetic actuator that utilizes light-driven ion channels or ion pumps for controlling the activity of excitable cells like channelrhodopsin, halorhodopsin, and bacteriorhodopsin [45], while the activation of cells was recorded through optogenetics sensors such as hybrid voltage sensors [46] and RCaMP sensors [47].

Brain function has a crucial role in the development of physiological activities. The ongoing discussion on overlapping neurobiological traits and psychiatric disorders such as depression and substance-related disorders embodied the importance of the human brain [48, 49]. Based on holism, which is one of the classical subjects of study in the philosophy of TCM [50], we can assume this whole brain as a subholism of the TCM theory system. Therefore, the investigation of optogenetics revealing how the brain works in neuroscience is under the holistic frame of TCM. Scientists transferred light-sensitive opsin into any chosen subpopulation of neurons in the brain by using genetic engineering techniques. Then, when light shines on the brain through fiberoptics or other light-guiding tools, only those neurons will be activated or inactivated [51] and the manifestation of brain function will be more intuitive and simple. Light-gated ion channels will be selective to the passage of cations or anions under different wavelengths of light stimulation, such as $\mathrm{Cl}^{-}$and $\mathrm{Na}^{+}$, which will cause changes in the membrane potential between both sides of the cell membrane to achieve selective excitation or inhibition. Channelrhodopsin (ChR) is one of the most classic genes of light-sensing genes [52]. When blue light hits it, it opens up a little hole and allows charged particles $\left(\mathrm{Na}^{+}\right)$to enter the eye-spot, allowing this eye-spot to have an electrical signal, thereby generating action potentials and making the neurons in a state of excitement (Figure 2). Halorhodopsin is an optogenetic tool that effectively inhibits neuronal activity [53]. When yellow light hits it, it lets other charged particles $\left(\mathrm{Cl}^{-}\right)$enter the membrane, which puts the neurons at resting potential, thereby maintaining a resting state (Figure 2). This means that TCM optogenetics can observe the neuronal activity of different populations and syndromes in the same encephalopathy and also monitor the effect of some neurons after the intervention of Chinese medicine.

4.2. The Properties of High Spatiotemporal Resolution. The spatiotemporal resolution, offered by optogenetics, is a huge asset for the precise manipulation of cellular processes [54]. It can also be used simultaneously at multiple wavelengths and locations to figure out the presence or activity of specific molecules [18]. Since Karl Deisseroth and his colleagues reported using light-gated cation channel channelrhodopsin to make neurons controllable by light in 2005 [55], the optogenetic approach has been gradually improved. Currently, researchers have found new opsins with more effective inhibition of neuronal activity, which are more sensitive to light stimulation and remain open for a long time [56].

How could the information precisely reach where researchers want it to go? Scientists took the light-sensitive DNA, put it into a gene vector, like a virus, and delivered it into the target cell. The targeted cell used its natural proteinmaking to fabricate those little light-sensitive proteins and installed them all over the cell and then have the cell which can be activated marked with light. The implications can be introduced into many fields, such as translation medicine [57] and regenerative medicine [58]. Even more amazing is that scientists devote themselves to a higher level optogenetic approach to explore the operation of aneural circuits. Multistepped optogenetics is the strategy to induce expression of the target gene using multi-irradiation to simultaneously explore the anatomy and behavioral functions of known and unknown neurons in vivo [59]. So far, the research of optogenetics has revealed some neural signal pathways and found many bonds between proteins, cells, tissues, and nerve functions [56].

The temporal precision and spatial resolution that optogenetics offers are unequaled by other available methods [60]. Based on the high spatiotemporal resolution, optogenetics will enable precise regulation in both time and space after TCM intervention, especially acupuncture. Although the positive effects of acupuncture have been gradually confirmed, they are still being questioned due to the lack of scientific basis for the specific cellular and molecular mechanisms [61-63]. This characteristic of the high spatiotemporal resolution will clarify the potential sites of action or pathways of acupuncture and even may monitor local physiologic changes in different acupuncture manipulations, needling depth, acupuncture locations at different times. 


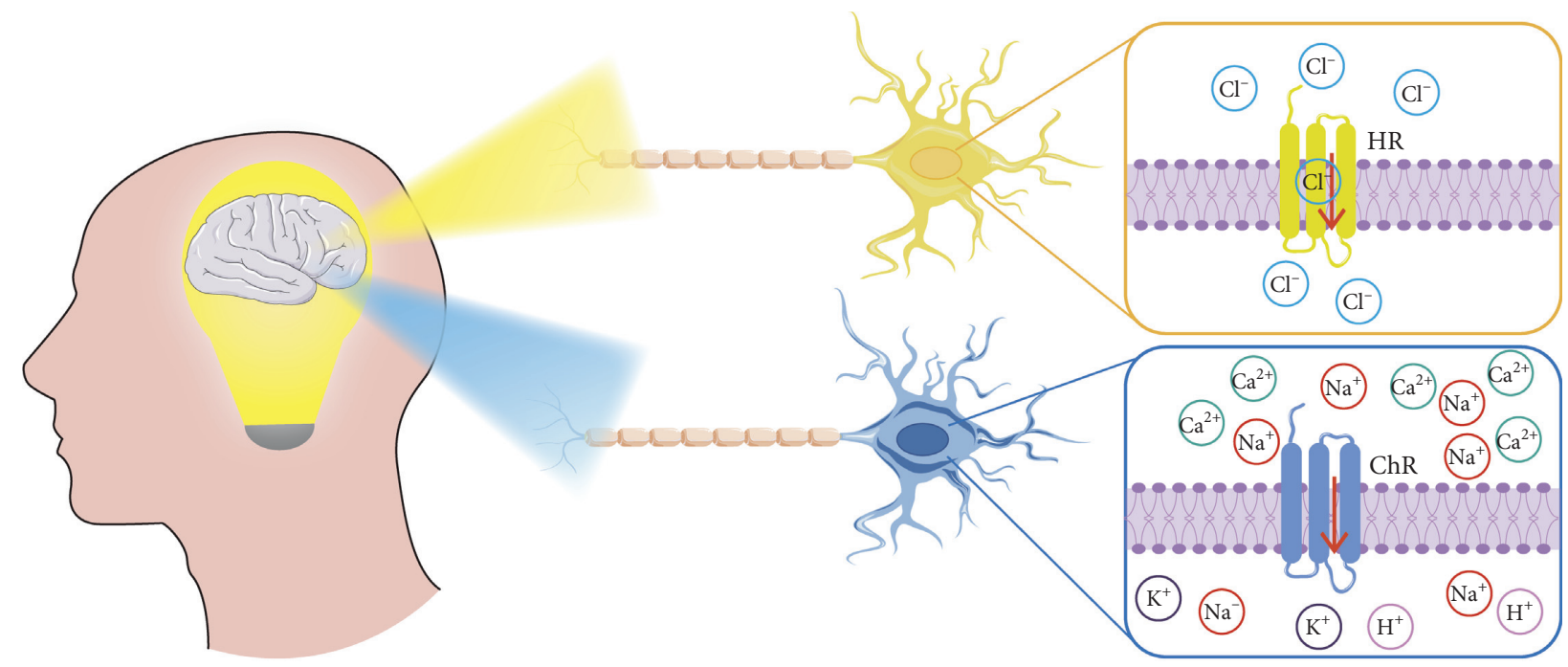

FIgURE 2: The fundamentals of the optogenetic process. ChR: channelrhodopsin; HR: halorhodopsin. Through genetic skill delivering the opsin into the cell of the model mouse brain, ChR and HR can convert light into electricity. When blue light hits it, it opens up a little hole and allows charged particles $\left(\mathrm{Na}^{+}\right)$to enter the eye-spot, allowing this eye-spot to have an electrical signal. When yellow light hits it, it lets other charged particles $\left(\mathrm{Cl}^{-}\right)$enter the membrane.

4.3. The Potential of Identifying the Different Zheng. TCM is a recognition system that involves the unceasing summary, induction, and extraction of the accumulated experience to prevent and treat the common diseases, while Zheng is the core concept of TCM theory. It is for diagnosis of disease states, the advance of treatment effect, and the strategy of individual treatment. The essence of TCM is, as it were, the differences between the Zhengs [64]. Zheng reacts to the type of pathological changes in a certain stage. It tends to reveal the nature of the disease integrally and truly. The classification of Zheng always depends on the intuition and experience of the physician trained in TCM theory [22]. Each Zheng of TCM will involve multiple anatomical tissues and systems in normal conditions. Hence, unclear and nonobjective are disadvantages of TCM syndrome differentiation. On the other hand, Zheng does have the basis of a long-term clinical and rich phenotype division; it indeed provides some right directions for treating the disease. This ultraprecise optogenetic approach is incorporated into a network-like structure of related cells to investigate the molecular basis underlying Zheng. Thus, it is able to determine how each of the Zheng has a cellular activity pathway to achieve the modernization and standardization of TCM and then offer some shreds of evidence [65]. For example, optogenetics may be utilized to observe the discharge of cells in the hypothalamus-pituitary-adrenal axis of kidney deficiency patients. Compared with healthy human, different functions of cells may exist, which have another expression of optogenetics.

Moreover, patients with the same disease might exhibit different Zhengs and should, therefore, receive different treatments. Conversely, patients with different diseases might exhibit the same Zheng and should, therefore, receive the same treatments [66]. Multiple diseases might share one "pattern" and be treated by the same herbal formula, whereas one disease might display several different "patterns" and be treated by multiple formulas [67]. An important application of optogenetics is to associate different functions of cells with phenotypic differences between groups of individuals to obtain phenotype-specific information. Ideally, TCM optogenetics could be served as a potential tool to discriminate against the Zheng of many diseases. In the contrasts of devitalization of heart yang and insufficiency of heart yin, we can turn different kinds of cells on and off through the circuit to find out which one contributes to certain functions and which one goes wrong in certain pathology. The changes in cell membrane potential may be different when applied with the optogenetic approach. For this idea, the TCM optogenetics will offer an evidence-based basis for understanding the foundation of Zheng, the relevance of TCM syndrome and effect, and the relationship between Chinese medical formulas and Zheng. The function of these light-sensitive cells might explain the potential mechanisms and foundations of a specific TCM Zheng (Table 2). Next, it could be promoted to the study of other TCM systems (the five internal organs and the six hollow organs).

\section{Potential Applications of TCM Optogenetics}

A series of optogenetic methods have been utilized to investigate diseases widely [68-75]. TCM optogenetics will play an unsubstituted role in the future. Hence, we explored potential future applications of TCM optogenetics.

5.1. The Study of Correlation in Zheng and Prescription. The syndrome differentiation of TCM is a process of dynamic change along with the evolution of the disease. In normal conditions, the disease will result in a novel Zheng with time, and the treatment will be changed following the Zheng. TCM optogenetics may diagnose Zheng quicker so 
TABLE 2: Summary of optogenetics may study of TCM Zheng.

\begin{tabular}{|c|c|c|c|}
\hline TCM Zheng & $\begin{array}{l}\text { Disease or } \\
\text { model }\end{array}$ & $\begin{array}{l}\text { Relevant } \\
\text { parts }\end{array}$ & Potential mechanism \\
\hline $\begin{array}{l}\text { Cold syndrome/heat } \\
\text { syndrome }\end{array}$ & Whole body & $\mathrm{PBMC}$ & $\begin{array}{l}\text { The cold syndrome might be caused by inhibition of cells, while the heat } \\
\text { syndrome might be caused by activation of cells. }\end{array}$ \\
\hline $\begin{array}{l}\text { Yin deficiency/Yang } \\
\text { deficiency }\end{array}$ & Whole body & Brain & $\begin{array}{l}\text { The Yin deficiency might be caused by inhibition of cells, while the Yang } \\
\text { deficiency might be caused by activation of cells. }\end{array}$ \\
\hline Blood stasis syndrome & $\mathrm{CHD}$ & PBMC & $\begin{array}{c}\text { Circuits, cells, parts, and pathways of the heart are turned on and off to } \\
\text { understand the pathologic index. }\end{array}$ \\
\hline Kidney deficiency & NS & $\begin{array}{l}\text { HPAT axis/ } \\
\text { brain }\end{array}$ & $\begin{array}{l}\text { Different cells in different brain regions are activated or inhibited to figure out } \\
\text { what is wrong with the cells and what is the pathologic circuit. }\end{array}$ \\
\hline Spleen deficiency & NS & Brain cortex & $\begin{array}{l}\text { For ultraprecise control of the cells, we need to reveal the specific neural circuit of } \\
\text { spleen deficiency. }\end{array}$ \\
\hline $\begin{array}{l}\text { Pulmonary-Qi } \\
\text { deficiency }\end{array}$ & COPD & $\mathrm{PBMC}$ & $\begin{array}{l}\text { A highly differential pathway of pulmonary cells is shown between pulmonary-qi } \\
\text { deficiency and healthy subjects. }\end{array}$ \\
\hline Liver-qi stagnation & Depression & Brain & $\begin{array}{l}\text { The synapse active pathway of central and peripheral 5-HT release is pursued to } \\
\text { expose specific brain circuit elements. }\end{array}$ \\
\hline
\end{tabular}

PBMC, peripheral blood mononuclear cell; CHD, coronary heart disease; HPAT, hypothalamus-pituitary-adrenal-thymus; NS, nonspecific disease; COPD, chronic obstructive pulmonary disease.

that the use of Chinese formulas may be more accurate and lead to excellent efficiency in the future. In one aspect, this postulate may offer optimization of using Chinese herbs. For example, Buyang Huanwu decoction (BYHWD), a TCM formula, has been recognized as a treatment for coronary heart diseases (CHD) with Qi deficiency and blood stasis syndrome in some studies. Clinical studies have indicated that $\mathrm{CHD}$ is associated with the elevation of many inflammatory biomarkers. However, the effects of BYHWD on inflammatory pathways in CHD with Qi deficiency and blood stasis syndrome are rarely known [76]. Optogenetics uses the genetic encoding of light sensitivity (by the expression of microbial opsins) to provide the capabilities for manipulation, recording, and controlling by light with cell specificity and high spatiotemporal resolution in CHD of Qi deficiency and blood stasis. Optogenetics aims to investigate the mechanisms of BYHWD affecting CHD with Qi deficiency and blood stasis syndrome and to confirm optimal dosages of each herb according to the function of the cellular network. If the blood stasis syndrome disappears (i.e., Qi deficiency of CHD), cardiomyocytes will have different optogenetics expression and then could be used to design other corresponding traditional Chinese prescriptions ( $\mathrm{Fu}$ fang).

In recent years, the exploration focusing on the Zheng and traditional Chinese decoction has become one of the hottest fields of modern TCM research [77]. Employing pharmacological and pharmaceutical sciences is crucial to discuss the efficiency and mechanism of the Chinese formulas in the different levels of prescription of herbs, location, composition, and the object of holistic, tissue, cell, and molecule. It is rare to build a scientific model between Zheng and Chinese decoction. And the biological connotation of one Chinese medicine formula is still unclear [78]. TCM optogenetics will identify target points of multiple Chinese herbal ingredients across the signs and symptoms and then consider this as a constellation or pattern, which is unique to each person $[28,79]$. The Zheng of depression, such as liverqi stagnation which results in depression of anger and distress according to TCM theory, can be relieved with an ancient TCM formula called Chaihu-Shugan-San (CSS) [80]. The optogenetic method can be applied to pursue the synapse active pathways of central and peripheral 5-HT release to expose the antidepressant cellular function network of CSS. More importantly, optogenetics can read messages quickly so that they can be synchronized with optical control. This can be achieved by recording "optical fibers" or the reporter proteins, which is a biological sensor of the fluorescent protein fused to the detection of proteins [81]. In this paradigm, the Zheng and Chinese formulas will be more consistent with the concept of evidence-based medicine.

\subsection{Reveal the Therapy Impact of TCM in Neurological} Disorders. With the rapid tendency of the aging society, the incidence of traumatic brain injury is rising sharply [82]. Human neurological disorders have an increasing threat to public health care. As a micro integral, the brain dominates almost all behaviors of our life. Recent years have seen molecular imaging, such as PET and fMRI, which revealed the functions of the whole human brain. These imaging techniques have been applied in TCM therapy for neurological disorders to assess noninvasively disease-specific cellular and molecular levels of living models in vivo [6]. However, the limitation of PET is the inherently low spatial resolution, thus little anatomical information [83]. The fMRI increases the temporal resolution but worsens the spatial resolution. Therefore, only imaging modality cannot provide all the information to comprehensively monitor the effects of TCM therapy in neurological disorders (such as Alzheimer's disease [6]).

Modulation of defined neural projections (the unique and most versatile features of optogenetics) is well aligned with altered function along pathways of neural communication (a core feature of psychiatric disease) [84]. That is crucial for optogenetic technology and psychiatry. TCM therapeutics, consistent with the small holism of the brain, 
has its special advantage over western medicine in treating neurological diseases $[85,86]$. It has the characteristics of multiple pathways and multiple targets [87]. With the basic function of these complex mechanisms still under study, the frontier technologies of TCM research are self-evident. Thus, we can observe some specific cells or even the whole brain by the optogenetics after TCM therapy. This may be a tool for real-time detection of TCM and to record the specific trajectories that we want to make explicit.

Furthermore, TCM therapy coherent with molecular imaging methodologies may currently enable researchers to image and manipulate activity from specific neurological disorders of cells with better contrast and resolution, higher speeds, and deeper into brain tissues [84]. One example is the multimodal fMRI platform that provides detailed brainwide network activation maps for the corpus callosumspecific optogenetic stimulation [88]. This new advance will bring a significant breakthrough in the exploration of TCM therapy.

5.3. Research on the Mechanisms of Acupuncture. Acupuncture is used to shift a person's unique "climate." The classic theory recognizes about 365 points, said to be located on 14 main channels (or meridians) connecting the body in a weblike interconnecting matrix. Fine needles are inserted into precisely defined specific points on the body to correct disruptions in harmony. Recent years have seen an increased interest in the use of acupoint stimulation to address the growing numbers of individuals suffering from pain, chronic or acute diseases, or psychological distress [89]. However, effective channels are not detectable by ordinary scientific methods. Although basic researches have begun to elucidate the mechanisms of action of acupuncture, promising results have emerged from clinical studies of acupuncture for emesis [90], low back pain and postoperative dental pain [91], acupuncture analgesia [92-94], and so on. Kim et al. [95] found that acupuncture effects on the methamphetamine- (METH-) induced changes were abolished by optogenetic inhibition of $\mathrm{CeA}$, indicating that the acupuncture effect was mediated through CeA neurons. This finding fills a critical gap in the effects of acupuncture on METH-induced behaviors and neural pathways. This provides strong evidence for studying the mechanism of acupuncture. The value and modernization of acupuncture are worth further studying [35]. Up to now, new technology such as fMRI is beginning to demonstrate that acupuncture has regionally specific, quantifiable effects on relevant structures of the human brain [17]. The efficacy of acupuncture on the outcome measures is still equivocal. Thus, optogenetics has investigative superiority to observe the causal role of intracellular biochemical signals in circuit and animal behavior in acupuncture therapy.

Numerous studies have demonstrated that the nervous system, neurotransmitters, endogenous substances, and Jing Luo (meridians) may respond to needling stimulation and electrical acupuncture [96]. Optogenetics may determine how acupuncture act on different cell types through different acupoint stimulations. In the recent acupuncture general anesthesia experiment, Chen et al. measured the depth of sedation by recording heart rate (HR), mean blood pressure (MAP), and NT index [97]. Through an optical sensor that transmits the cell function signal and photoelectrode, which precisely controls target cells at a millisecond scale, optogenetics will quickly capture the targeted cell type with fire activity and then precisely form a specific mechanism pathway of the acupoint. Moreover, the optogenetic approach could timely and precisely feed back the long-term effect of cell signal. These can accurately unveil how acupuncture generates the efficiency of general anesthesia. Similarly, optogenetics may shed light on the CNS mechanism of acupuncture analgesia and form a basis for future investigations of endogenous pain modulation circuits in the human brain.

With the development of high-tech acupuncture, laser acupuncture may be the rudiments of the integration of acupuncture and optogenetics in the future. As of August 2018, according to statistics from Gerhard Litscher, there were more than 900 research papers on laser acupuncture [98]. The top four countries were China, Austria, ISLAMed.Uni Graz, USA, and Germany It can be seen that laser acupuncture was extensively researched around the world. Gerhard Litscher reported the results of the first use of blue laser acupuncture needle in 2009 [99]. Compared with subjects $(5 \%-10 \%)$ stimulated with red or infrared laser, $80 \%$ of subjects stimulated with blue laser acupuncture needle had an immediate deQi-sensation [99]. In addition, the temperature and flux at the Hegu point also increased significantly [99]. Moreover, a series of studies revealed that violet laser stimulation $(405 \mathrm{~nm})$ can increase the blood flow velocity [100], increase vascular effects on microcirculation at acupoint Dazhui [101], change in skin surface temperature distributions [102], significantly decrease in aortic pulse wave velocity [103], and mediate HR $[104,105]$.

The existence of laser provides the possibility of activating light-sensitive genes. Therefore, we can introduce some light-sensitive genes into the target cells of the tissues where the acupoints are located, such as skin cells, cardiomyocytes, and brain endothelial cells. When laser acupuncture is performed, these light-sensitive genes are activated to track the precise mechanisms of action of the acupuncture points. If we perform laser acupuncture on multiple acupoints on a certain meridian, we can even verify the traveling routes of the meridian, which exerts beneficial effects in revealing the mechanism of traditional acupuncture. Xiong et al. used a miniature blue light-emitting diode $(470 \mathrm{~nm})$ to stimulate $\mathrm{S} 1$ activity in ChR2 transgenic mouse and indicated that optogenetic stimulation normalized cortical hyperexcitability through changing neuronal membrane properties and reducing the frequency of excitatory postsynaptic events [106]. However, ChR2 transgenic mice provide a powerful method for optogenetic research of the nervous system but reduced excitability and responsiveness of reticulospinal tracts [107]. Therefore, if we use this model to conduct optogenetic acupuncture experiments, we must consider the adverse effects of this potential factor on the experimental results. 
5.4. Combining Optogenetics with Other Technologies. Personalized medicine is significant for it refers to the right treatment for the right individual at the right time in the healthcare world. TCM is gaining increasing attention with the emergence of personalized medicine, characterized by treatment based on syndrome or Zheng differentiation. TCM has the potential to diminish the incidence of adverse drug reactions, eliminate invalid therapy, improve the efficacy of treatments, and ultimately achieve optimal health outcomes [108]. The future trend of applying optogenetics alone or integrated with other technique paradigms, such as omics and chemical analysis, aims to achieve personalized medicine of TCM.

The generation and development of disease are relevant to the structural and functional disorders of disturbed cells and the unbalance of whole body function. Optogenetics provides a basis for linking behavioral functions with cellular and circuit-level activity patterns [109]. Computer imaging or analysis leads to the utilization of optogenetics and obtains the complex target network of disease. Moreover, metabolomics, a powerful tool for understanding and resolving disease-related pathologies, will realize the holistic manipulation of individual diagnostics and therapeutics $[110,111]$. Therefore, the combination of the optogenetic approach and metabolomics would do much to help personalized medicine. Besides, the adoption of systematic optogenetic perturbation and metabolomics may explain the multitarget, holistic, and dynamic effects of the individual traditional Chinese prescription and gain the optimum personalized treatment system. In the future, TCM optogenetics must be combined with more technologies to provide a powerful help to explore the mystery of TCM.

\section{Conclusion}

TCM, as a holistic approach to health, attempts to bring the body, mind, and spirit into harmony. It is an essential part of the healthcare system in several Asian countries, but it is considered a complementary or alternative medical system in most Western countries [112]. The key issue is that the actual value of TCM has not been fully recognized worldwide due to a lack of scientific approaches to its study [113]. During recent decades, the research of optogenetics has been given wide attention by putting light convert to electricity just within a few milliseconds or seconds so that we can understand what necessary role cells play in the circuits in which they are embedded. Approaches to further understand the dynamics of TCM and its targets will likely be developed and may provide deeper insights into the mechanisms for TCM and facilitate its standardization and modernization. Optogenetics is a reductionist method and can also achieve the systematic study of Zheng, herbal formulas, the effect and mechanisms of TCM. Moreover, the combination of optogenetics and other methods will have a profound impact on TCM research. Although TCM optogenetics is still a hypothesis, such a novel approach will initiate new directions and lead to a probable revolution in the modernization of TCM. It also contributes new insights into the current TCM field.
Posed an ever technique of TCM, optogenetics will help uncover the mystery of TCM and increase its application.

\section{Conflicts of Interest}

The authors declare that they have no conflicts of interest.

\section{Authors' Contributions}

Yang Wang designed this review article. Wei-Kang Luo and Dan Zhou wrote the main manuscript text. Wei-Kang Luo, Jia-Miao Liu, Yang Hang, Zhao-Yu Yang, Yao Wu, and Liang-Lin Zhang revised the paper and were responsible for drawing and sorting out tables. All authors reviewed the manuscript.

\section{Acknowledgments}

This work was funded by a grant from the National Natural Science Foundation of China (Nos. 81973665, 81673719, and 81803948) and the Project of Inheritance Studio for National Famous Experts of Traditional Chinese Medicine (No. [2018]134).

\section{References}

[1] P. Drasar and J. Moravcova, "Recent advances in analysis of Chinese medical plants and traditional medicines," Journal of Chromatography B Analytical Technologies in the Biomedical and Life Sciences, vol. 812, no. 1-2, pp. 3-21, 2004.

[2] F. Y. Fung and Y. C. Linn, "Developing traditional chinese medicine in the era of evidence-based medicine: current evidences and challenges," Evidence-Based Complementary and Alternative Medicine, vol. 2015, Article ID 425037, 2015.

[3] K. Chan, X.-Y. Hu, V. Razmovski-Naumovski, and N. Robinson, "Challenges and opportunities of integrating traditional Chinese medicine into mainstream medicine: a review of the current situation," European Journal of Integrative Medicine, vol. 7, no. 1, pp. 67-75, 2015.

[4] M. J. Leach, "Profile of the complementary and alternative medicine workforce across Australia, New Zealand, Canada, United States and United Kingdom," Complementary Therapies in Medicine, vol. 21, no. 4, pp. 364-378, 2013.

[5] Z. Xing, Z. Xia, W. Peng et al., "Xuefu Zhuyu decoction, a traditional Chinese medicine, provides neuroprotection in a rat model of traumatic brain injury via an anti-inflammatory pathway," Scientific Reports, vol. 6, Article ID 20040, 2016.

[6] Z. Wang, H. Wan, J. Li, H. Zhang, and M. Tian, "Molecular imaging in traditional Chinese medicine therapy for neurological diseases," Biomed Research International, vol. 2013, Article ID 608430, 2013.

[7] J. Liu, K. Sun, C. Zheng et al., "Pathway as a pharmacological target for herbal medicines: an investigation from reduning injection," PLoS One, vol. 10, no. 4, Article ID e123109, 2015.

[8] X. Liang, H. Li, and S. Li, "A novel network pharmacology approach to analyse traditional herbal formulae: the LiuWei-Di-Huang pill as a case study," Mol. BioSyst. vol. 10, no. 5, pp. 1014-1022, 2014.

[9] S. Lukman, Y. He, and S.-C. Hui, "Computational methods for traditional Chinese medicine: a survey," Computer Methods and Programs in Biomedicine, vol. 88, no. 3, pp. 283-294, 2007. 
[10] T. Liu, J. Zhou, H. Cui et al., "Quantitative proteomic analysis of intracerebral hemorrhage in rats with a focus on brain energy metabolism," Brain and Behavior, vol. 8, no. 11, Article ID e1130, 2018.

[11] Y. Zhao, S. Nie, M. Yi et al., "UPLC-QTOF/MS-based metabolomics analysis of plasma reveals an effect of Xue-FuZhu-Yu capsules on blood-stasis syndrome in CHD rats," Journal of Ethnopharmacology, vol. 241, Article ID 111908, 2019.

[12] A. R. Adamantidis, F. Zhang, L. de Lecea, and K. Deisseroth, "Optogenetics: opsins and optical interfaces in neuroscience," Cold Spring Harb Protoc, vol. 2014, no. 8, pp. 815-822, 2014.

[13] Y. Farhatnia, "Channelrhodopsins: visual regeneration and neural activation by a light switch," New Biotechnology, vol. 30, no. 5, pp. 461-474, 2013.

[14] Insights of the decade, "Stepping away from the trees for a look at the forest. Introduction," Science, vol. 330, no. 6011, pp. 1612-1613, 2010.

[15] K. Deisseroth, “Optogenetics," Nature Methods, vol. 8, no. 1, pp. 26-29, 2011.

[16] J. Li, H. Wan, H. Zhang, and M. Tian, "Current applications of molecular imaging and luminescence-based techniques in traditional Chinese medicine," Journal of Ethnopharmacology, vol. 137, no. 1, pp. 16-26, 2011.

[17] T. He, W. Zhu, S.-Q. Du et al., "Neural mechanisms of acupuncture as revealed by fMRI studies," Autonomic Neuroscience, vol. 190, pp. 1-9, 2015.

[18] M. Yang, J. Zou, H. Zhu et al., "Paris saponin II inhibits human ovarian cancer cell-induced angiogenesis by modulating NF- $\kappa \mathrm{B}$ signaling," Oncology Reports, vol. 33, no. 5, pp. 2190-2198, 2015.

[19] W. L. Wang, S. Y. Sheu, Y. S. Chen et al., "Evaluating the bone tissue regeneration capability of the Chinese herbal decoction Danggui Buxue Tang from a molecular biology perspective," Biomed Research International, vol. 2014, Article ID 853234, , 2014.

[20] L. T. Ngo, J. I. Okogun, and W. R. Folk, "21st century natural product research and drug development and traditional medicines," Natural Product Reports, vol. 30, no. 4, pp. 584-592, 2013.

[21] S. Chen, J. Xu, C. Liu et al., "Genome sequence of the model medicinal mushroom Ganoderma lucidum," Nature Communications, vol. 3, p. 913, 2012.

[22] P. Wang and Z. Chen, "Traditional Chinese medicine ZHENG and Omics convergence: a systems approach to post-genomics medicine in a global world," OMICS: A Journal of Integrative Biology, vol. 17, no. 9, pp. 451-459, 2013.

[23] Y. Zhong, J. Luo, T. Tang et al., "Exploring pharmacological mechanisms of Xuefu Zhuyu decoction in the treatment of traumatic brain injury via a network pharmacology approach," Evidence-Based Complementary and Alternative Medicine, vol. 2018, Article ID 8916938, 2018.

[24] S. Li and B. Zhang, "Traditional Chinese medicine network pharmacology: theory, methodology and application," Chinese Journal of Natural Medicines, vol. 11, no. 2, pp. 110-120, 2013.

[25] C. Y. Chen, “TCM Database@Taiwan: the world's largest traditional Chinese medicine database for drug screening in silico," PLoS One, vol. 6, no. 1, Article ID e15939, 2011.

[26] H. Xu, Y. Zhang, Y. Lei et al., "A systems biology-based approach to uncovering the molecular mechanisms underlying the effects of dragon's blood tablet in colitis, involving the integration of chemical analysis, ADME prediction, and network pharmacology," PLoS One, vol. 9, no. 7, Article ID e101432, 2014.

[27] M. Ishtiaq, M. Maqbool, T. Hussain, S. Azam, and Y. Wang, "Leaf proteome analysis of clematis chinensis: a traditional chinese medicine (TCM) by two-dimensional electrophoresis technique," Pakistan Journal of Botany, vol. 46, no. 3, pp. 789-801, 2014.

[28] P. Gu and H. Chen, "Modern bioinformatics meets traditional Chinese medicine," Briefings in Bioinformatics, vol. 15, no. 6, pp. 984-1003, 2014.

[29] Y. Liu and N. Feng, "Nanocarriers for the delivery of active ingredients and fractions extracted from natural products used in traditional Chinese medicine (TCM)," Advances in Colloid and Interface Science, vol. 221, pp. 60-76, 2015.

[30] I. R. Bell, M. Koithan, and D. Pincus, "Methodological implications of nonlinear dynamical systems models for whole systems of complementary and alternative medicine," Forschende Komplementärmedizin/Research in Complementary Medicine, vol. 19, no. s1, pp. 15-21, 2012.

[31] K.-C. Lan and G. Litscher, "Robot-controlled acupuncturean innovative step towards modernization of the ancient traditional medical treatment method," Medicines, vol. 6, no. 3 , p. $87,2019$.

[32] G. Litscher, "Ten years evidence-based high-tech acupuncture part 3: a short review of animal experiments," EvidenceBased Complementary and Alternative Medicine, vol. 7, no. 2, pp. 151-155, 2010.

[33] J. B. G. Silva, "About acupuncture and electroacupuncture," Anesthesiology, vol. 121, no. 3, p. 662, 2014.

[34] Z. Liu, Y. Liu, H. Xu et al., "Effect of electroacupuncture on urinary leakage among women with stress urinary incontinence," JAMA, vol. 317, no. 24, pp. 2493-2501, 2017.

[35] XY. Gao, K. Liu, B. Zhu, and G. Litscher, "Sino-European transcontinental basic and clinical high-tech acupuncture studies-part 1: auricular acupuncture increases heart rate variability in anesthetized rats," Evidence-Based Complementary and Alternative Medicine, vol. 2012, Article ID 817378, 2012.

[36] G. Litscher, G. Cheng, W. Cheng et al., "Sino-European transcontinental basic and clinical high-tech acupuncture studies-part 2: acute stimulation effects on heart rate and its variability in patients with insomnia," Evidence-Based Complementary and Alternative Medicine, vol. 2012, Article ID 916085, 2012.

[37] XY. Gao, G. Litscher, K. Liu, and B. Zhu, "Sino-European transcontinental basic and clinical high-tech acupuncture studies-part 3: violet laser stimulation in anesthetized rats," Evidence-Based Complementary and Alternative Medicine, vol. 2012, Article ID 402590, 2012.

[38] G. Litscher, LP. Wang, L. Wang, CZ. Liu, and XM. Wang, "Sino-European transcontinental basic and clinical high-tech acupuncture studies-part 4: "fire of life" analysis of heart rate variability during acupuncture in clinical studies," EvidenceBased Complementary and Alternative Medicine, vol. 2012, Article ID 153480, 2012.

[39] Y. Jiang, B. David, P. Tu, and Y. Barbin, "Recent analytical approaches in quality control of traditional Chinese medicines-a review," Analytica Chimica Acta, vol. 657, no. 1, pp. 9-18, 2010.

[40] F. Gao, Y. Hu, G. Fang et al., "Recent developments in the field of the determination of constituents of TCMs in body fluids of animals and human," Journal of Pharmaceutical and Biomedical Analysis, vol. 87, pp. 241-260, 2014. 
[41] O. J. Abilez, J. Wong, R. Prakash, K. Deisseroth, C. K. Zarins, and E. Kuhl, "Multiscale computational models for optogenetic control of cardiac function," Biophysical Journal, vol. 101, no. 6, pp. 1326-1334, 2011.

[42] M. Häusser, "Optogenetics: the age of light," Nature Methods, vol. 11, no. 10, pp. 1012-1014, 2014.

[43] D. Chaudhury, J. J. Walsh, A. K. Friedman et al., "Rapid regulation of depression-related behaviours by control of midbrain dopamine neurons," Nature, vol. 493, no. 7433, pp. 532-536, 2013.

[44] G. P. Dugué, W. Akemann, and T. Knöpfel, "A comprehensive concept of optogenetics," Progress in Brain Research, vol. 196, pp. 1-28, 2012.

[45] A. Okazaki, M. Takahashi, N. Toyoda, and S. Takagi, "Optical silencing of $C$. elegans cells with light-driven proton pumps," Methods, vol. 68, no. 3, pp. 425-430, 2014.

[46] N. Ghitani, P. O. Bayguinov, Y. Ma, and M. B. Jackson, "Single-trial imaging of spikes and synaptic potentials in single neurons in brain slices with genetically encoded hybrid voltage sensor," Journal of Neurophysiology, vol. 113, no. 4, pp. 1249-1259, 2015.

[47] J. Akerboom, C. N. Carreras, L. Tian et al., "Genetically encoded calcium indicators for multi-color neural activity imaging and combination with optogenetics," Frontiers in Molecular Neuroscience, vol. 6, p. 2, 2013.

[48] S. Daniele, E. Da Pozzo, E. Zappelli, and C. Martini, "Trazodone treatment protects neuronal-like cells from inflammatory insult by inhibiting NF- $\kappa \mathrm{B}, \mathrm{p} 38$ and JNK," Cellular Signalling, vol. 27, no. 8, pp. 1609-1629, 2015.

[49] S. Aou, T. Inoue, T. Fujimoto et al., "Chemical impacts on higher brain functions," in International Congress Series, H. Nakagawa, K. Ishii, and H. Miyamoto, Eds., vol. 1269pp. 101-104, 2004.

[50] X. Pan, R. Valerdi, and R. Kang, "Systems thinking: a comparison between Chinese and western approaches," in Procedia Computer Science, C. Paredis, C. Bishop, and D. Bodner, Eds., vol. 16pp. 1027-1035, 2013.

[51] S. C. P. Williams and K. Deisseroth, "Optogenetics," Proceedings of the National Academy of Sciences, vol. 110, no. 41, p. 16287, 2013.

[52] K. Deisseroth and P. Hegemann, "The form and function of channelrhodopsin," Science, vol. 357, no. 6356, p. 5544, 2017.

[53] K. M. Tye, R. Prakash, S.-Y. Kim et al., "Amygdala circuitry mediating reversible and bidirectional control of anxiety," Nature, vol. 471, no. 7338, pp. 358-362, 2011.

[54] W. W. D. Mühlhäuser, A. Fischer, W. Weber, and G. Radziwill, "Optogenetics-bringing light into the darkness of mammalian signal transduction," Biochimica et Biophysica Acta (BBA)-Molecular Cell Research, vol. 1864, no. 2, pp. 280-292, 2017.

[55] E. S. Boyden, "Interview with Edward S. Boyden," Trends in Neurosciences, vol. 36, no. 1, pp. 1-2, 2013.

[56] J. Wietek, J. S. Wiegert, N. Adeishvili et al., "Conversion of channelrhodopsin into a light-gated chloride channel," Science, vol. 344, no. 6182, pp. 409-412, 2014.

[57] J. Cao, M. Arha, C. Sudrik, D. V. Schaffer, and R. S. Kane, "Bidirectional regulation of mRNA translation in mammalian cells by using PUF domains," Angewandte Chemie International Edition, vol. 53, no. 19, pp. 4900-4904, 2014.

[58] M. A. Zarbin, T. Arlow, and R. Ritch, "Regenerative nanomedicine for vision restoration," Mayo Clinic Proceedings, vol. 88, no. 12, pp. 1480-1490, 2013.
[59] M. Itoh, T. Yamamoto, Y. Nakajima, and K. Hatta, "Multistepped optogenetics connects neurons and behavior," Current Biology, vol. 24, no. 24, pp. R1155-R1156, 2014.

[60] S. Jarvis and SR. Schultz, "Prospects for optogenetic augmentation of brain function," Frontiers in Systems Neuroscience, vol. 9, p. 157, 2015.

[61] A. S. Cheifetz, R. Gianotti, R. Luber, and P. R. Gibson, "Complementary and alternative medicines used by patients with inflammatory bowel diseases," Gastroenterology, vol. 152, no. 2, pp. 415-429, 2017.

[62] A. Meakins, “Acupuncture: what's the point?” British Journal of Sports Medicine, vol. 51, no. 6, p. 484, 2017.

[63] J. Marchant, "Acupuncture in cancer study reignites debate about controversial technique," Nature, vol. 552, no. 7684, pp. 157-158, 2017.

[64] S.-m. Li, H. Xu, and K.-j. Chen, "The diagnostic criteria of blood-stasis syndrome: considerations for standardization of pattern identification," Chinese Journal of Integrative Medicine, vol. 20, no. 7, pp. 483-489, 2014.

[65] S. B. Su, W. Jia, A. Lu, and S. Li, "Evidence-based ZHENG: a traditional Chinese medicine syndrome 2013," EvidenceBased Complementary and Alternative Medicine, vol. 2014, Article ID 484201, , 2014.

[66] J. Dai, S. Sun, J. Peng et al., "Exploration of macro-micro biomarkers for dampness-heat syndrome differentiation in different diseases," Evidence-Based Complementary and Alternative Medicine, vol. 2013, Article ID 706762, 2013.

[67] W. Y. Jiang, "Therapeutic wisdom in traditional Chinese medicine: a perspective from modern science," Discovery Medicine, vol. 5, no. 29, pp. 455-461, 2005.

[68] A. R. Adamantidis, F. Zhang, A. M. Aravanis, K. Deisseroth, and L. de Lecea, "Neural substrates of awakening probed with optogenetic control of hypocretin neurons," Nature, vol. 450, no. 7168, pp. 420-424, 2007.

[69] P. Albert, "Light up your life: optogenetics for depression?" Journal of Psychiatry \& Neuroscience, vol. 39, no. 1, pp. 3-5, 2014.

[70] J. N. Bentley, C. Chestek, W. C. Stacey, and P. G. Patil, "Optogenetics in epilepsy," Neurosurgical Focus, vol. 34, no. 6, 2013.

[71] J. Tonnesen, C. L. Parish, A. T. Sorensen et al., "Functional integration of grafted neural stem cell-derived dopaminergic neurons monitored by optogenetics in an in vitro Parkinson model," PLoS One, vol. 6, no. 3, Article ID e17560, 2011.

[72] F. H. Do-Monte, K. Quiñones-Laracuente, and G. J. Quirk, "A temporal shift in the circuits mediating retrieval of fear memory," Nature, vol. 519, no. 7544, pp. 460-463, 2015.

[73] A. Zahedi, K. Defea, and I. Ethell, "Optogenetics to target actin-mediated synaptic loss in alzheimer's," in Proceedings of SPIE, S. K. Mohanty and N. V. Thakor, Eds., vol. 8586, 2013.

[74] D. R. Sparta, J. Smithuis, A. M. Stamatakis et al., "Inhibition of projections from the basolateral amygdala to the entorhinal cortex disrupts the acquisition of contextual fear," Frontiers in Behavioral Neuroscience, vol. 8, p. 129, 2014.

[75] L. M. Yager, A. F. Garcia, A. M. Wunsch, and S. M. Ferguson, "The ins and outs of the striatum: role in drug addiction," Neuroscience, vol. 301, pp. 529-541, 2015.

[76] H. Zhang, W.-R. Wang, R. Lin et al., "Buyang Huanwu decoction ameliorates coronary heart disease with Qi deficiency and blood stasis syndrome by reducing CRP and CD40 in rats," Journal of Ethnopharmacology, vol. 130, no. 1, pp. 98-102, 2010. 
[77] M. Jiang, C. Lu, C. Zhang et al., "Syndrome differentiation in modern research of traditional Chinese medicine," Journal of Ethnopharmacology, vol. 140, no. 3, pp. 634-642, 2012.

[78] M. Zhao, Q. Zhou, W. Ma, and D. Q. Wei, "Exploring the ligand-protein networks in traditional chinese medicine: current databases, methods, and applications," EvidenceBased Complementary and Alternative Medicine, vol. 2013, Article ID 806072, 2013.

[79] J. Dai, S. Sun, H. Cao et al., "Applications of new technologies and new methods in ZHENG differentiation," EvidenceBased Complementary and Alternative Medicine, vol. 2012, Article ID 298014, 2012.

[80] Y. Wang, R. Fan, and X. Huang, "Meta-analysis of the clinical effectiveness of traditional Chinese medicine formula Chaihu-Shugan-San in depression," Journal of Ethnopharmacology, vol. 141, no. 2, pp. 571-577, 2012.

[81] S. Usherenko, H. Stibbe, M. Musco, L. O. Essen, E. A. Kostina, and C. Taxis, "Photo-sensitive degron variants for tuning protein stability by light," BMC Systems Biology, vol. 8, p. 128, 2014.

[82] A. I. Maas, N. Stocchetti, and R. Bullock, "Moderate and severe traumatic brain injury in adults," The Lancet Neurology, vol. 7, no. 8, pp. 728-741, 2008.

[83] G. Wolf and N. Abolmaali, "Preclinical molecular imaging using PET and MRI," Molecular Imaging in Oncology, vol. 187, pp. 257-310, 2013.

[84] K. Deisseroth, "Optogenetics and psychiatry: applications, challenges, and opportunities," Biological Psychiatry, vol. 71, no. 12, pp. 1030-1032, 2012.

[85] Z.-L. Ren and P.-P. Zuo, "Neural regeneration: role of traditional Chinese medicine in neurological diseases treatment," Journal of Pharmacological Sciences, vol. 120, no. 3, pp. 139-145, 2012.

[86] C. Gao and J. Shen, "Metabolic factors and adult neurogenesis: impacts of Chinese herbal medicine on brain repair in neurological diseases," International Review of Neurobiology, vol. 135, pp. 117-147, 2017.

[87] Y. Cao, G. Yao, Y. Sheng et al., "JinQi Jiangtang tablet regulates gut microbiota and improve insulin sensitivity in type 2 diabetes mice," Journal of Diabetes Research, vol. 2019, Article ID 1872134, , 2019.

[88] Y. Chen, F. Sobczak, P. Pais-Roldán, C. Schwarz, AP. Koretsky, and X. Yu, "Mapping the brain-wide network effects by optogenetic activation of the corpus callosum," Cerebral Cortex, vol. 30, no. 11, p. 164, 2020.

[89] S. A. Gilomen and C. W. Lee, "The efficacy of acupoint stimulation in the treatment of psychological distress: a meta-analysis," Journal of Behavior Therapy and Experimental Psychiatry, vol. 48, pp. 140-148, 2015.

[90] K. Rithirangsriroj, T. Manchana, and L. Akkayagorn, "Efficacy of acupuncture in prevention of delayed chemotherapy induced nausea and vomiting in gynecologic cancer patients," Gynecologic Oncology, vol. 136, no. 1, pp. 82-86, 2015.

[91] R. Staud and D. D. Price, "Mechanisms of acupuncture analgesia for clinical and experimental pain," Expert Review of Neurotherapeutics, vol. 6, no. 5, pp. 661-667, 2006.

[92] QY. He, Z. Shen, LJ. She, JQ. Fang, and XM. Shao, "Feasibility of joint application of techniques of optogenetics and neuroelectrophysiology to research of acupuncture analgesia," Zhen Ci Yan Jiu, vol. 43, no. 8, pp. 476-479, 2018.

[93] S. A. Ismail, H. M. Atef, M. E. Abuelnaga, and H. M. Midan, "Unilateral acupuncture reduces postoperative pain scores in children undergoing adenotonsillectomy: a randomized controlled trial," Journal of Pain Research, vol. 14, pp. 273283, 2021.

[94] M. M. S. Trento, A. O. O. Moré, E. C. W. Duarte, and D. F. Martins, "Peripheral receptors and neuromediators involved in the antihyperalgesic effects of acupuncture: a state-of-the-art review," Pflügers Archive, Springer, Berlin, Germany, 2021.

[95] M. S. Kim, Y. Fan, S. M. Lee et al., "Role of the central amygdala in acupuncture inhibition of methamphetamineinduced behaviors in rats," Addiction Biology, vol. 26, no. 1, Article ID e12862, 2021.

[96] S.-X. Ma, "Neurobiology of acupuncture: toward CAM," Evidence-Based Complementary and Alternative Medicine, vol. 1, no. 1, pp. 41-47, 2004.

[97] Y. Chen, H. Zhang, Y. Tang, and J. Shu, "Impact of bilateral ST36 and PC6 electroacupuncture on the depth of sedation in general anaesthesia," Acupuncture in Medicine, vol. 33, no. 2, pp. 103-109, 2015.

[98] G. Litscher, "Laser acupuncture research: China, Austria, and other countries-update 2018," Medicines, vol. 5, no. 3, p. 92, 2018.

[99] G. Litscher, "Modernization of traditional acupuncture using multimodal computer-based high-tech methods-recent results of blue laser and teleacupuncture from the Medical University of Graz," Journal of Acupuncture and Meridian Studies, vol. 2, no. 3, pp. 202-209, 2009.

[100] G. Litscher, T. Huang, L. Wang, and W. Zhang, "Violet laser acupuncture-part 1: effects on brain circulation," Journal of Acupuncture and Meridian Studies, vol. 3, no. 4, pp. 255-259, 2010.

[101] L. Wang, T. Huang, W. Zhang, and G. Litscher, "Violet laser acupuncture-part 2: effects on peripheral microcirculation," Journal of Acupuncture and Meridian Studies, vol. 4, no. 1, pp. 24-28, 2011.

[102] G. Litscher, L. Wang, T. Huang, and W. Zhang, "Violet laser acupuncture-part 3: pilot study of potential effects on temperature distribution," Journal of Acupuncture and Meridian Studies, vol. 4, no. 3, pp. 164-167, 2011.

[103] G. Litscher, L. Wang, I. Gaischek, and X.-Y. Gao, "Violet laser acupuncture-part 4: acute effects on human arterial stiffness and wave reflection," Journal of Acupuncture and Meridian Studies, vol. 4, no. 3, pp. 168-174, 2011.

[104] G. Litscher, Z. Xie, L. Wang, and I. Gaischek, "Blue 405 nm laser light mediates heart rate-investigations at the acupoint Neiguan (Pe.6) in Chinese adults," North American Journal of Medical Sciences, vol. 1, no. 5, pp. 226-231, 2009.

[105] W. He, D. Wedig, L. Wang, I. Gaischek, and G. Litscher, "Violet laser acupuncture-part 5: an investigation of different stimulation frequencies on heart rate and variability," Journal of Acupuncture and Meridian Studies, vol. 5, no. 6, pp. 290-294, 2012.

[106] W. Xiong, X. Ping, MS. Ripsch et al., "Enhancing excitatory activity of somatosensory cortex alleviates neuropathic pain through regulating homeostatic plasticity," Scientific Reports, vol. 7, no. 1, Article ID 12743, 2017.

[107] W. Wu, W. Xiong, P. Zhang et al., "Increased threshold of short-latency motor evoked potentials in transgenic mice expressing channelrhodopsin-2," PLoS One, vol. 12, no. 5, Article ID e0178803, 2017.

[108] A. Zhang, H. Sun, P. Wang, Y. Han, and X. Wang, "Future perspectives of personalized medicine in traditional Chinese medicine: a systems biology approach," Complementary Therapies in Medicine, vol. 20, no. 1-2, pp. 93-99, 2012. 
[109] Y. Mei and F. Zhang, "Molecular tools and approaches for optogenetics," Biological Psychiatry, vol. 71, no. 12, pp. 1033-1038, 2012.

[110] A. Zhang, H. Sun, Z. Wang, W. Sun, P. Wang, and X. Wang, "Metabolomics: towards understanding traditional Chinese medicine," Planta Medica, vol. 76, no. 17, pp. 2026-2035, 2010.

[111] T. Li, Y. Zhong, T. Tang et al., "Formononetin induces vasorelaxation in rat thoracic aorta via regulation of the PI3K/PTEN/Akt signaling pathway," Drug Design, Development and Therapy, vol. 12, pp. 3675-3684, 2018.

[112] E. Chan, M. Tan, J. Xin, S. Sudarsanam, and D. E. Johnson, "Interactions between traditional Chinese medicines and Western therapeutics," Current Opinion in Drug Discovery \& Development, vol. 13, no. 1, pp. 50-65, 2010.

[113] H. Cao, A. Zhang, H. Zhang, H. Sun, and X. Wang, "The application of metabolomics in traditional Chinese medicine opens up a dialogue between Chinese and Western medicine," Phytotherapy Research, vol. 29, no. 2, pp. 159-166, 2015. 